\title{
Reden wir nicht über Glück
}

\section{Lebensqualität als dienstliche und persönliche Gestaltungsaufgabe}

WOLF RAINER WENDT

Prof. Dr. Wolf Rainer Wendt ist Vorsitzender der Deutschen Gesellschaft für Care und Case Management sowie Honorarprofessor der Eberhard Karls Universität Tübingen. Er ist Mitbegründer und langjähriger Vorsitzender der Deutschen Gesellschaft für Soziale Arbeit. Seit über drei Jahrzehnten ist er Mitglied im Beirat der Blätter der Wohlfahrtspflege.

E-Mail prof.dr.wendt@googlemail. com

Für den Einzelnen ergibt sich seine persönliche Lebensqualität aus vielen Faktoren und Momenten - seines Wohnens, seiner Berufsarbeit, seiner Beziehungen, seiner Mobilität, in der Kleidung, im guten Essen. Soziale Arbeit ist in diesem Sinne für Glück nicht zuständig. Sie kann jedoch zur "Wohlfahrt" des Menschen beratend, unterstützend und intervenierend beitragen, indem sie Veränderungen in seinen objektiven und subjektiven Lebensverhältnissen bewirkt.

Die Soziale Arbeit hat ihre Mühe damit, dass ihre Klientel im Leben einigermaßen zurechtkommt - Eltern mit ihren Kindern, Jugendliche zwischen Schule und Beruf, Suchtkranke mit ihrer Abhängigkeit, Menschen mit einer Behinderung im Alltag, chronisch Kranke mit ihren Belastungen, Wohnungslose auf der Straße.

Für sie ist Lebensqualität zunächst eine abstrakte Kategorie; die Sorgen sind andere und die Probleme drängen, aber wie so oft kommen gesellschaftliche Erwartungen, die an Dienstleistungen gestellt werden, auch auf die professionell Helfenden zu und sie müssen sich mit ihnen auseinandersetzen. Was und in welchen Bezügen trägt Soziale Arbeit zur Lebensqualität bei?

\section{Vom Wohlstand zur Lebensqualität}

Nachdem sich in der zweiten Hälfte des 20. Jahrhunderts in den entwickelten Ländern der materielle Wohlstand verbreitet hatte, wurde ein Ungenügen an ihm deutlich. Statt von nötiger Güterausstattung begann man, von Lebensqualität zu reden. Die Menge konsumierbarer Waren garantiert ein Wohlergehen des Menschen nicht.
Was aber zur Lebensqualität gehört, erwies sich als komplex und schwierig zu bestimmen. Gewöhnlich sieht man sie in der Verfügung über Ressourcen gegeben. So gehören materielle Sicherheit durch hinreichendes Einkommen, Einbindung ins Arbeitsleben, Gesundheit, befriedigende soziale Beziehungen, angenehmes Wohnen, Mobilität, Freizeitmöglichkeiten und Teilhabe an der Kultur der Gemeinschaft zur Lebensqualität. Man hat herausgefunden, dass sich objektive Lebensbedingungen und subjektives Wohlbefinden nicht decken, aber in einer kollektiv und individuell einzuschätzenden Lebensqualität aneinander gebunden sind. (Glatzer/Zapf 1984) Auf persönlicher Ebene wird Ausgewogenheit gesucht, insbesondere in der Balance von Leben und Arbeit, von Familie und Beruf.

Mit der Zeit ist Lebensqualität auf jeder Ebene der Politik bis zur Weltbank und Weltgesundheitsorganisation zum Thema geworden. Gleichzeitig wird sie individuell eingefordert. Personenbezogen hat der Diskurs über Lebensqualität seit einigen Jahren eine Zuspitzung dahingehend erfahren, indem nun Glück gefragt ist. In die Feuilletons zog die Glücksforschung ein; es wird nach Indikatoren des Wohlbefindens gesucht 
(OECD 2011). Wie zufrieden Deutschland ist, sagt uns ein Glücksatlas (Kücher/Raffelhüschen 2011); eine Menge Ratgeber zum Glücklichsein sind auf dem Buchmarkt zu haben, und im fernen Bhutan ist das Bruttosozialglück zur Maßgabe staatlichen Handelns geworden (vgl. Kasten »Die vier Säulen des Bruttonationalglücks und das gute Leben $\ll$ ).

\section{Lebensgestaltung stationär und mit ambulanter Beihilfe}

Im Feld der Sozialen Arbeit hat Lebensqualität primär seine Bedeutung für Einrichtungen, in denen sich Menschen dauernd aufhalten. In einem Heim oder einer anderen Form stationärer Unterbringung und Versorgung soll den Bewohnern eine hinreichende Lebensqualität geboten werden. Diese wird nicht von ihnen bestimmt und hängt nicht von ihrer eigenen Lebensführung $a b$. Es ist die ganze Ausstattung der Einrichtung, die Wohnqualität verheißt; das Personal bietet Betreuungsqualität; die Heimatmosphäre wird von der Führung des Hauses und von internen Beziehungen geprägt; Teilhabe am gemeinschaftlichen Leben bedeutet Sozialqualität; das Angebot an diversen Aktivitäten bietet eine Erlebnisqualität. Maßgebend sollen die Vorstellungen und Wünsche der Klientel sein. Pflegebedürftige Menschen schätzen andere Faktoren der Versorgung, zum Beispiel in der Sicherheit und in der vom Haus gebotenen Entlastung, als Menschen mit einer geistigen Behinderung, für die bei aller Teilhabe die Wahrung von Autonomie und Respekt wesentlich sind (Schäfers 2008).

Außerhalb von Einrichtungen begegnen Sozialprofessionelle ihrer Klientel in deren eigener Situation und selbstständiger Lebensführung. Ambulante Dienste begleiten die Menschen, nehmen zwar Einfluss auf sie, bestimmen aber in der Regel nicht über sie. Einzelne Hilfen betreffen die individuelle Lebensqualität nicht direkt, können jedoch zu ihr beitragen. Anders bei Veranstaltungen, die professionell organisiert und durchgeführt werden und sich für die Teilnehmer als eine Ressource guten Ergehens gestalten lassen: Gruppentreffen und andere gemeinschaftliche Unternehmungen, Bildungsmaßnahmen, Erholungsangebote. Die Dienstleistung hat ihre eigene Gestalt und das Programm beschreibt überindividuell, wofür es gut sein soll. Dagegen lässt sich im Einzelfall das qualitative Resultat personenbezogener Hilfen nicht von den Helfenden festlegen.

\section{Der Horizont individueller Erfahrung}

Was der Einzelne erstrebt und wie er leben will, wird in der Beratung und in der Unterstützung bei Problemen nicht unbedingt erörtert, gar umfassend geklärt. Schließlich ist Lebensqualität auch kein Anlass für den Einzelnen, sich Hilfe zu holen. Für ihn ergibt sich seine persönliche Lebensqualität aus vielen einzelnen Faktoren und Momenten seines Wohnens, seiner Berufsarbeit, seiner freundschaftlichen, sexuellen und familiären Beziehungen, seiner Mobilität, von Events in der Freizeit, in der Kleidung, die er trägt oder gerne tragen möchte, guten Essens usw.

Damit mögen Momente seines Lebensglücks verbunden sein. Gewöhnlich kann man sich bei ihnen in der Bewältigung dessen, was täglich ansteht, kaum aufhalten. Und in der allfälligen Misere des Lebens suchen viele Menschen ein Zufallsglück im Spiel und nicht wenige das Glück des Genusses im Rausch. Beziehungen missglücken, Vorhaben scheitern, Erwartungen an das Leben werden enttäuscht, der Alltag befriedigt ohne professionelle Beteiligung sozial gearbeitet.

Professionell Helfende sind gut beraten, sich bei einem Klienten zu erkundigen, wie er selber im Alltag zurechtkommt, Unterstützung und Hemmnisse erfährt, seine Lebensaufgaben sieht und sie zu meistern sucht. Es geht um seine Wohlfahrt als eine Erstreckung menschlichen Ergehens in der Art und dem $\mathrm{Maß}$, in dem es gedeihlich verläuft und vollzogen wird. So ist "Wohlfahrt" dem Wortsinn nach zu verstehen. Wir können Wohlfahrt als die Verlaufsform von Lebensqualität begreifen, wie sie sich bei einer Person in eigener Aktivität unter förderlichen Bedingungen wie in günstigen oder ungünstigen Verhältnissen einstellt. An ihnen lässt sich arbeiten.

Im Sozialstaat ist "Wohlfahrt « einer verbreiteten Auffassung nach zu einem öffentlichen Angebot geworden und hat den Charakter eines lieferbaren Gutes angenommen. "Von der Wohlfahrt leben « - amerikanisch »to be on welfare« - beschreibt den Status eines Sozialhilfeempfängers. Nehmen wir dagegen Klienten nicht als passive, sondern als aktive, selbständige und selbstverantwortliche Partner in der Bearbeitung ihrer Probleme wahr, wird das individuelle Wohl zu einer Zielstellung, die der Selbstsorge und eigener Lebensbewältigung einer Person oder Familie obliegt und die darin zu unterstützen ist.

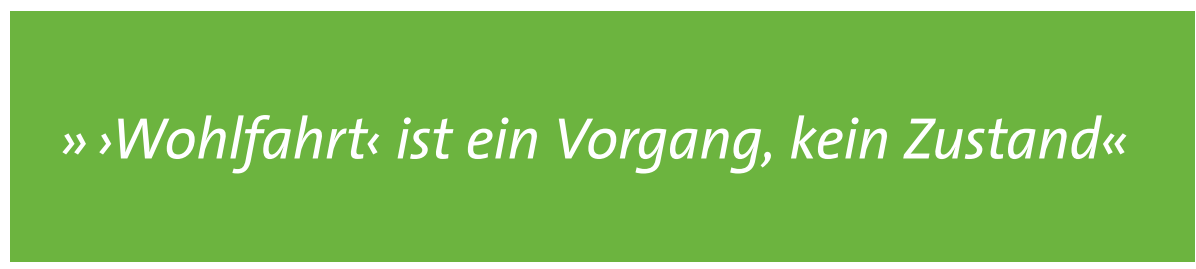

nicht. Materielle Versorgung und sozialberufliche Interventionen kommen dagegen nicht an und ersetzen kein persönlich erfahrenes Lebensglück.

Helfen und zurüsten kann Soziale Arbeit zur Bewältigung von Belastungen, zur Lebensgestaltung unter gegebenen Umständen und zur selbstständigen Lebensführung. Damit sind nicht Teilaspekte des Zurechtkommens eines Menschen oder einer Familie angesprochen, sondern gemeint ist das Kontinuum eigenen Sorgens auf dem Weg des persönlichen und auch gemeinsamen Ergehens. An ihm und den es bedingenden Verhältnissen wird informell auch
Weil ein gutes Ergehen sich als ein Vorgang in der Zeit vollzieht, es biografisch zu ermessen und mit der Perspektive eines Gelingens versehen ist, braucht es auch Zeit. Punktuelle Interventionen allein bringen Wohlfahrt nicht zuwege. Lebensqualität ist deshalb unter dem Blickwinkel nötiger Versorgung in erster Linie bei chronischer Erkrankung, bei Behinderung oder andauernder Pflege gefragt. Es handelt sich um eine andauernde Gestaltungsaufgabe.

Bei Kindern und Heranwachsenden ist Wohlfahrt eine Perspektive, die man in der familiären Erziehung, unter Bildungs-, Berufsfindungs- und Qualifi- 

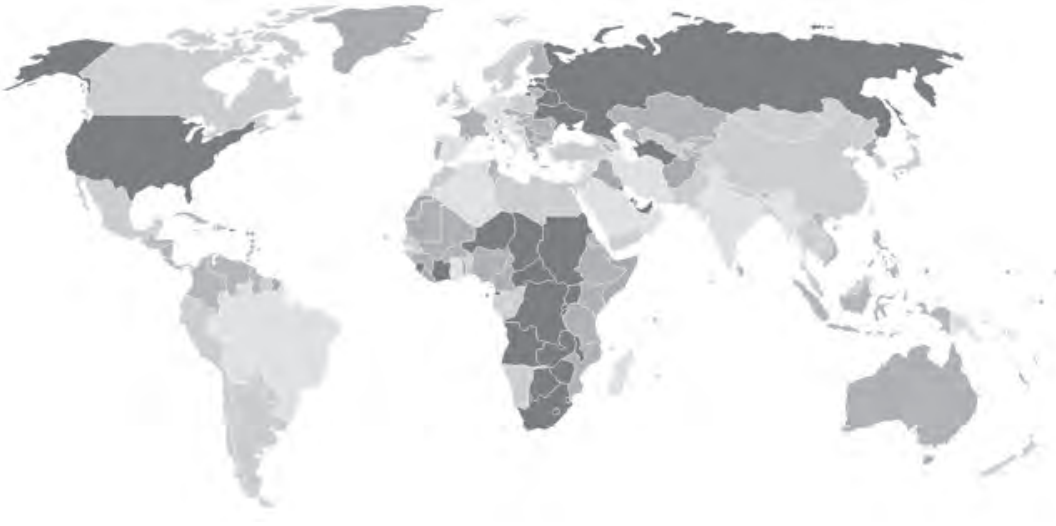

Der »Happy Planet Index« ist ein Versuch, weltweit Lebensqualität abzubilden. Dazu werden Werte für Lebenszufriedenheit, Lebenserwartung und ökologisches Verhalten kombiniert. Der Index wurde Im jahre 2006 von der New Economics Foundation in Zusammenarbeit mit Friends of the Earth publiziert. Die grau dargestellten Länder haben die besten Werte, die dunkelgrauen die schlechtesten.

\section{Die vier Säulen des Bruttonationalglücks und das gute Leben}

"Bruttonationalglück“ (BNG) ist der Versuch, den Lebensstandard in breit gestreuter, humanistischer und psychologischer Weise zu definieren und somit dem herkömmlichen Bruttonationaleinkommen, einem ausschließlich durch Geldflüsse bestimmten Maß, einen ganzheitlicheren Bezugsrahmen gegenüberzustellen. Der Ausdruck wurde 1979 von Jigme Singye Wangchuck, dem König von Bhutan geprägt, in Entgegnung auf ein Interview mit einem indischen Journalisten, der sich nach dem Bruttoinlandsprodukt von Bhutan erkundigt hatte. Der König wollte damit zum Ausdruck bringen, dass er sich einer Wirtschaftsentwicklung verpflichtet fühle, die Bhutans einzigartiger Kultur und ihren buddhistischen Werten gerecht werde. Bhutan hat zu diesem Zweck mit der Kommission für das Bruttonationalglück eine Staatskommission eingesetzt. Während konventionelle Entwicklungsmodelle das Wirtschaftswachstum zum herausragenden Kriterium politischen Handelns machen, nimmt die Idee des Bruttonationalglücks an, dass eine ausgewogene und nachhaltige Entwicklung der Gesellschaft nur im Zusammenspiel von materiellen, kulturellen und spirituellen Schritten geschehen kann, die einander ergänzen und bestärken. Die vier Säulen des Bruttonationalglücks sind:

- die Förderung einer sozial gerechten Gesellschafts- und Wirtschaftsentwicklung

- Bewahrung und Förderung kultureller Werte

- Schutz der Umwelt und

- gute Regierungs- und Verwaltungsstrukturen.

Bruttonationalglück lässt sich nur schwer objektiv messen und unterliegt einer Reihe von subjektiven Werturteilen. Dies ist bei üblichen Wirtschafts- und Sozialmodellen gleichermaßen der Fall. Da die entscheidende Frage ist, wer die Eckpunkte des Bezugsrahmens definiert, kommt der Art und Weise der politischen Willensbildung insbesondere der Diskussion im Rahmen der Verfassungsgebung von 2008 in Bhutan große Bedeutung zu. Einen ähnlichen Weg gingen Ecuador und Bolivien mit der Verankerung des indigenen Prinzips des "Sumak kawsay" ("gutes Leben", span. "buen vivir») in der ecuadorianischen Verfassung von 2008 und der bolivianischen Verfassung von 2009.

In einem vom New Economic Foundation's Centre for Well-Being in London erstellten "Happy Planet Index", der Lebenserwartung und Zufriedenheit der Bevölkerung in Relation zu den konsumierten Gütern setzt, belegt Vanuatu den ersten Platz, gefolgt von Kolumbien. Die USA stehen in dieser Liste auf Platz 150, noch hinter Entwicklungsländern wie Burkina Faso. Der Index wurde von Robert Stavins, einem Wirtschaftswissenschaftler der Harvard University, kritisiert, weil er lediglich die ideologische Voreingenommenheit seiner Autoren widerspiegele.

Quelle: www.de.wikipedia.org zierungsaspekten und komplementär oder kompensatorisch in der Jugendhilfe im Blick haben kann. Wie »wohl« dem jungen Menschen dabei ist, steht auf einem anderen Blatt. Kinder sind noch nicht soweit, sich aus guten Gründen selber für ein bestimmtes Leben zu entscheiden. $\mathrm{Ob}$ es gelingt, stellt sich später heraus.

Man bleibt zu den Qualitäten seines Daseins persönlich unterwegs und nähert sich einem Gelingen in eigener Lebensführung nur an. Der Glückszustand eines »Es ist erreicht « gehört weder zur individuellen noch zur gemeinschaftlichen Wohlfahrt.

\section{Chancen bekommen und eine Perspektive eröffnen}

Soziale Arbeit ist für Glück nicht zuständig. Sie kann auch das Unglück nicht beheben, das einen Menschen getroffen hat. Soziale Arbeit trägt zum Ergehen des Einzelnen beratend, unterstützend und intervenierend bei, indem sie Veränderungen in seinen objektiven und subjektiven Lebensverhältnissen bewirkt. Klienten werden, soweit sie nicht ohnehin motiviert sind, zu diesem Wandel herangezogen. Ihnen können Verwirklichungsmöglichkeiten geboten werden, die sie sich aber auch zueigen machen müssen (vgl. Volkert 2005). Dass aus vorhandenen oder zu erschlieBenden Chancen etwas wird, bedarf häufig wiederum einer zurüstenden, bildenden Arbeit.

Das Wohl zum Beispiel einer alleinerziehenden Mutter mit mehreren Kindern, ohne berufliche Ausbildung, verschuldet und auf Hartz IV angewiesen, ist in ihrer Lage und in steter Absprache mit ihr vielseitig zu besorgen: in Hinblick auf das schulische Vorankommen der Kinder, auf soziale Kontakte, Beratung in Erziehungs- und Gesundheitsfragen, auf die eine oder andere Qualifizierungsmaßnahme, auf finanzielle Hilfen und schrittweise Entschuldung, usw. Präferenzen sind auszumachen, bei deren Abarbeitung nach und nach eine Besserung erlebt wird. Die Erfahrung des Vorankommens und eines Gewinns an Handlungsspielraum tut wohl.

Persönliche Lebensqualität ist keine statische Eigenschaft, sondern will entworfen und tätig hervorgebracht werden. Menschen leben mit einer Perspektive. Deshalb wird die Lebensqualität 
eines Studenten, der wenig Mittel hat, in einem kleinen Zimmer wohnt und in seinen Kräften voll ausgelastet ist, von ihm deutlich positiver wahrgenommen als die eines Arbeitslosen mit mehr Mitteln und größerer Wohnung, aber subjektiv ohne Aussicht auf Änderung. Ihm geht es schlecht; in seiner Lage kann keine Rede von Wohlfahrt sein. Sie hat einen dynamischen Charakter - und der fehlt bei Arbeitslosigkeit. Dagegen ist die Wohlfahrt des Studierenden absehbar und insoweit schon vorhanden. Zu ihr gehört eine »optimale
Beanspruchung« (Laszlo 2008), die den Menschen voranbringt.

Beanspruchung ist keine Last und Not, solange sie mit Handlungsfreiheit (»agency«) einhergeht. In Belangen der Lebensqualität besteht die Gestaltungsaufgabe Sozialer Arbeit in der Eröffnung und Beibringung von Verwirklichungsmöglichkeiten, die eine Person für sich und das Leben, das sie führt, realisieren kann - inbegriffen als Bildungsaufgabe die Befähigung, solche Chancen tatsächlich wahrnehmen zu können.

\section{Literatur}

\section{Bellebaum, Alfred/Hettlage, Robert (Hg.):}

Glück hat viele Gesichter. Annäherungen an eine gekonnte Lebensführung. VS, Wiesbaden 2010.

Glatzer, Wolfgang/Zapf, Wolfgang (Hg.): Lebensqualität in der Bundesrepublik. Objektive Lebensbedingungen und subjektives Wohlbefinden. Campus, Frankfurt am Main 1984.

Kücher, Renate/Raffelhüschen, Bernd: Glücksatlas, Deutschland 2011. Knaus, München 2011. Laszlo, Herbert: Glück und Wirtschaft. Infothek, Wien 2008.

Layard, Richard: Die glückliche Gesellschaft. Kurswechsel für Politik und Wirtschaft. Campus, Frankfurt am Main 2005.

OECD (ed.): How's Life? Measuring well-being. OECD Publishing, Paris 2011.

Schäfers, Markus: Lebensqualität aus Nutzersicht. VS, Wiesbaden 2008

Volkert, Jürgen (Hg.): Armut und Reichtum an Verwirklichungschancen. VS, Wiesbaden 2005.

\section{UNSERE PROGRAMME FÜR DIE ZUKUNFT: NACHHALTIG SOZIAL INVESTIEREN}

\section{Energieeffizienz bringt Sie voran}

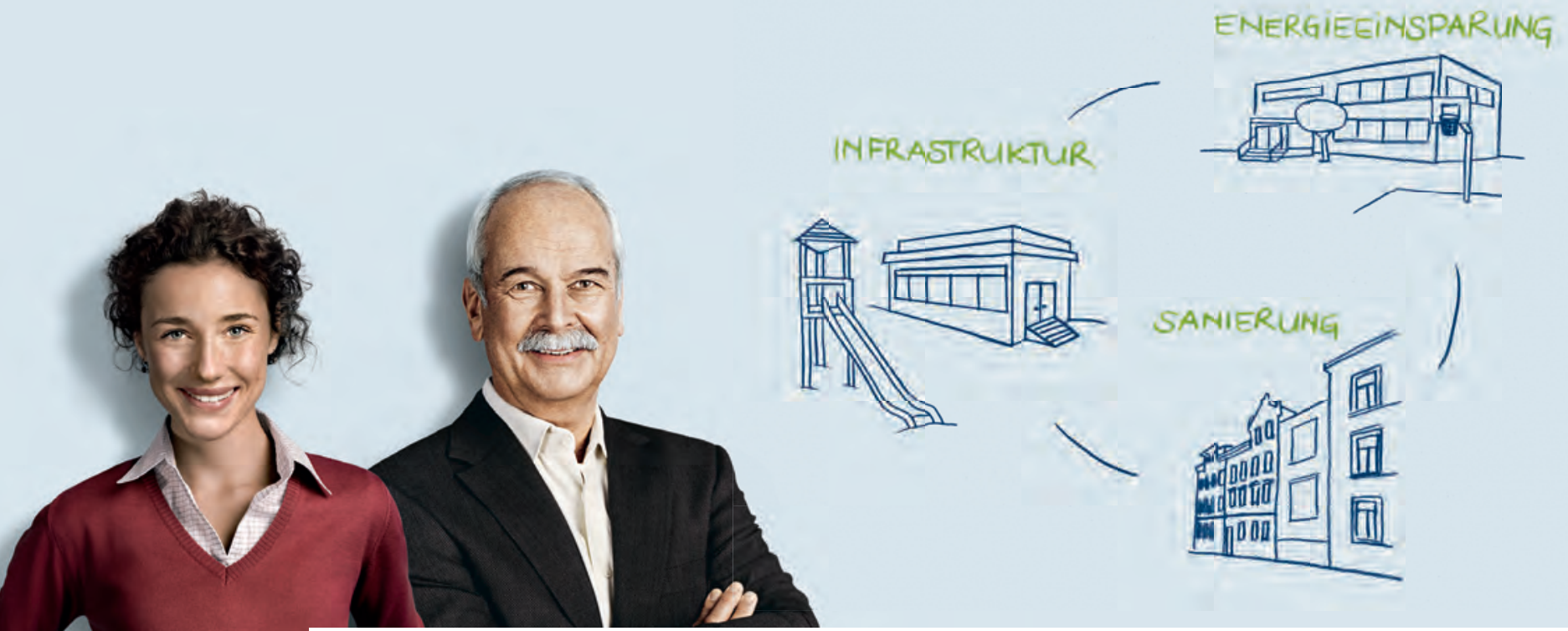

Mit den Umweltprogrammen der KfW können Sie Ihre kommunale und soziale Infrastruktur nachhaltig verbessern. Wir sind Ihr Partner, wenn Sie als gemeinnützige Organisation Kindergärten, Schulen, Krankenhäuser, Altenpflegeeinrichtungen, Verwaltungsgebäude oder andere Gebäude energetisch sanieren möchten. Wir beraten Sie gerne.

www.kfw.de/infrastruktur_infocenter@kfw.de 